\title{
Molecular Analysis of Eight American Type Culture Collection Gonococcal Strains by Neisseria gonorrhoeae Multiantigen Sequence Typing and PorB Sequence Typing
}

Yousun Chung ${ }^{1}$, Minje Han ${ }^{1}$, Ji-Young Park ${ }^{1,2}$, Sora Kang ${ }^{1}$, Inhee Kim $^{1}$, Jung A Park ${ }^{1}$, and Jae-Seok Kim ${ }^{1,2}$ ${ }^{1}$ Department of Laboratory Medicine, Kangdong Sacred Heart Hospital, Seoul; ${ }^{2}$ Department of Laboratory Medicine, Hallym University College of Medicine, Chuncheon, Korea

Corresponding author: Jae-Seok Kim Department of Laboratory Medicine, Kangdong Sacred Heart Hopsital, 150 Seonganro, Gangdong-gu, Seoul 05355, Korea

Tel: $+82-2-2224-2327$

Fax: +82-2-2224-2214

E-mail: jaeseokcp@gmail.com
Background: Molecular epidemiological typing of Neisseria gonorrhoeae is crucial for monitoring the spread of resistant strains. As reference strains can be used for laboratory internal quality control, we genetically characterised the American Type Culture Collection (ATCC) gonococcal strains by Neisseria gonorrhoeae multiantigen sequence typing (NGMAST) and porB sequence typing using public multilocus sequence typing (PubMLST).

Methods: Eight ATCC gonococcal reference strains (ATCC 19424, ATCC 31426, ATCC 35541, ATCC 43069, ATCC 43070, ATCC 49226, ATCC 49926, and ATCC 49981) from Culti-Loops (Thermo Fisher Scientific, USA) were cultured. After DNA extraction, porB and $t b p B$ were amplified and sequenced. Sequence types (STs) and allele numbers were each determined by NG-MAST (http://www.ng-mast.net) and porB sequence typing using PubMLST (http://pubmlst.org/neisseria/porB/).

Results: ATCC 19424 was identified as ST 266 by NG-MAST, and as Allele 946 by PubMLST. ATCC31426 was assigned a novel ST by NG-MAST, and was assigned Allele 958 with $1.2 \%$ mismatch by PubMLST. ATCC 35541 was identified as ST 12 by NG-MAST, and as Allele 624 by PubMLST. ATCC 43069 and ATCC 43070 were both identified as ST 681 by NGMAST, and as Allele 984 by PubMLST. ATCC 49226 was identified as ST 1572 by NG-MAST, and as Allele 2110 by PubMLST. ATCC 49926 and ATCC 49981 were both identified as ST 16496 by NG-MAST, and as Allele 928 by PubMLST.

Conclusions: The ST data obtained for ATCC gonococcal reference strains by NG-MAST and porB sequence typing using PubMLST can be used for quality assurance of molecular epidemiological typing in clinical microbiological laboratories.

(J Lab Med Qual Assur 2019;41:24-28)

Key Words: Neisseria gonorrhoeae, American Type Culture Collection, Reference, porB, $t b p B$, Neisseria gonorrhoeae multiantigen sequence typing, Public multilocus sequence typing

Received December 19, 2018, Revision received January 25, 2019, Accepted January 30, 2019

\section{INTRODUCTION}

Neisseria gonorrhoeae is a species of Gram-negative diplococci that causes gonorrhoea. Gonorrhoea is the second most common bacterial sexually transmitted infection, which leads to substantial morbidity and economic costs worldwide. There are an estimated 78 million new cases of gonorrhoea among adults aged 15 to 49 years worldwide [1-3]. Molecular epidemiological typing of $N$. gonorrhoeae is crucial for monitoring the spread of resistant strains, especially in light of the globally increasing antimicrobial resistance of this pathogen [4,5]. 


\section{Journal of LABORATORY MEDICINE and QUALITY ASSURANCE}

\section{Yousun Chung et al $\bullet$ Molecular Typing of ATCC N. gonorrhoeae}

Among the various methodologies used in epidemiological studies, $N$. gonorrhoeae multiantigen sequence typing (NG-MAST) and public multilocus sequence typing (PubMLST) approaches have been widely adopted for molecular typing [6-17].

NG-MAST is an online public database based on the analysis of sequences of internal fragments of two hypervariable genes, $\operatorname{por} B$ and $t b p B$, which encode superficial gonococcus antigens [5]. The NG-MAST analysis results are presented in the form of discrete allele numbers and sequence types (STs) for each isolate. This approach has been used globally to study prevalent strain genotypes, their transmission in sexual networks and core groups, and to describe distributions of resistant strains [6-11]. PubMLST is also an online public database, which originated as part of the development of the multilocus sequence typing (MLST) approach to the characterisation of bacterial strains in 1998 [12]. Databases for Neisseria spp. are available for the MLST approach using seven genes (abcZ, adk, aroE, fumC, gdh, $p d h C$, and pgm) and are also available for single gene approaches which target porA, porB, fetA, fHbp, nhba, and nadA. There have been many epidemiological studies to characterise Neisseria spp. using PubMLST [11,13-17].

Microbial reference strains are essential in diagnostic or research studies to verify the reliability of the test results and can be used as internal quality control for quality assurance. The American Type Culture Collection (ATCC) is one of the most commonly used microbial reference resources worldwide. In the current study, we genetically characterised and evaluated the stabilities of ATCC $N$. gonorrhoeae reference strains by NG-MAST and porB sequence typing using PubMLST.

\section{MATERIALS AND METHODS}

\section{Culture of $N$. gonorrhoeae reference strains}

Eight ATCC gonococcal reference strains (ATCC 19424, ATCC 31426, ATCC 35541, ATCC 43069, ATCC 43070, ATCC 49226, ATCC 49926, and ATCC 49981) were analysed. ATCC 49226 is the strain designated by the Clinical and Laboratory Standards Institute- designated strain for antimicrobial susceptibility testing of $N$. gonorrhoeae [18]. Culture was performed 5 times with a 1-month interval for each reference strain with five individually packaged Culti-Loops (Thermo Fisher Scientific, Waltham, MA, USA) from February to June 2018. Culti-Loops were stored in $4^{\circ} \mathrm{C}$ according to the instructions of the manufacturer. N. gonorrhoeae strains were cultured on chocolate agar plates (Shin Yang Chemical, Seoul, Korea) and incubated for 48 hours at $36^{\circ} \mathrm{C}$ under $5 \% \mathrm{CO}_{2}$. A single colony was subcultured once prior to DNA preparation.

\section{DNA extraction}

Bacterial suspensions $\left(\mathrm{OD}_{540}=1.0-2.0\right)$ were prepared in $0.17 \mathrm{~mol} / \mathrm{L}$ phosphate-buffered saline. The bacteria were pelleted by centrifugation at $2,000 \times \mathrm{g}$ for 5 minutes, washed once, resuspended in phosphate-buffered saline, and boiled for 5 minutes. The lysate was centrifuged at $2,000 \times g$ for 5 minutes, and the supernatant was used for DNA extraction. Bacterial DNA was extracted using the QIAamp DNA Mini Kit (Qiagen, Hilden, Germany) using a standard protocol. DNA was extracted individually from colonies isolated from each loop to confirm reproducibility.

\section{Amplification and sequencing of porB and tbp $B$}

Polymerase chain reaction (PCR) and sequencing were performed as previously described [5]. Primers for porB (5'-CAAGAAGACCTCGGCAA-3' [forward) and 5'CCGACAACCACTTGGT-3' [reverse]) were designed using conserved sequences encoding pre-loop 2 and preloop 8 of the porin protein, to amplify a 737-bp fragment. Primers for tbpB (5'-CGTTGTCGGCAGCGCGAAAAC-3' 〔forward] and 5'-TTCATCGGTGCGCTCGCCTTG-3' [reverse]) were designed using conserved sequences to amplify a 589-bp fragment. The PCR reaction involved an initial 4-minute denaturation at $95^{\circ} \mathrm{C}$, followed by 25 cycles of denaturation at $95^{\circ} \mathrm{C}$ for 30 seconds, annealing at $58^{\circ} \mathrm{C}$ (porB) or $69^{\circ} \mathrm{C}(t b p B)$ for 30 seconds, and elongation at $72^{\circ} \mathrm{C}$ for 1 minute, with a final 10 -minute extension at $72^{\circ} \mathrm{C}$ and cooling to $4^{\circ} \mathrm{C}$. The size of the amplified products was confirmed by electrophoresis. DNA was extracted from the gel and then sequenced using a BigDye Terminator v3.1 


\section{Journal of LABORATORY MEDICINE and QUALITY ASSURANCE}

\section{Yousun Chung et al • Molecular Typing of ATCC N. gonorrhoeae}

Cycle Sequencing Kit (Applied Biosystems, Foster City, CA, USA) on an ABI 3730xl Genetic Analyzer (Applied Biosystems).

\section{Molecular analysis by NG-MAST and PorB sequence typing}

For NG-MAST, an online public database (http:// www.ng-mast.net) was used to assign allele numbers to the porB and $t b p B$ genes and STs for each strain. For porB sequence typing, an online public database (http:// pubmlst.org/neisseria/porB/) was used to type the porB gene and assign allele numbers to each strain.

\section{RESULTS}

\section{Recovery of $N$. gonorrhoeae reference strains from Culti-Loops}

ATCC 19424, ATCC 43069, ATCC 49226, and ATCC 49981 were recovered from all five cultures. ATCC 31426, ATCC 35541, and ATCC 43070 were recovered from four out of five cultures. ATCC 49926 was recovered from three

Table 1. Molecular characteristics of ATCC Neisseria gonorrhoeae reference strains $(n=8)$

\begin{tabular}{|c|c|c|c|c|c|}
\hline \multirow{3}{*}{$\begin{array}{l}\text { ATCC } \\
\text { strain }\end{array}$} & \multirow{3}{*}{ Recovery $^{*}$} & \multicolumn{3}{|c|}{ NG-MAST } & \multirow{3}{*}{$\begin{array}{l}\text { Pub- } \\
\text { MLST } \\
\text { Allele } \\
\text { (porB }\end{array}$} \\
\hline & & \multicolumn{2}{|c|}{ Allele } & \multirow{2}{*}{ ST } & \\
\hline & & por $B$ & $t b p B$ & & \\
\hline 19424 & $5 / 5$ & 201 & 90 & 266 & 946 \\
\hline 31426 & $4 / 5$ & $8,442^{\dagger}$ & 362 & New & $958^{*}$ \\
\hline 35541 & $4 / 5$ & 8 & 5 & 12 & 624 \\
\hline 43069 & $5 / 5$ & 103 & 196 & 681 & 984 \\
\hline 43070 & $4 / 5$ & 103 & 196 & 681 & 984 \\
\hline 49226 & $5 / 5$ & 1,022 & 6 & 1,572 & 2110 \\
\hline 49926 & $3 / 5$ & 90 & 350 & 16,496 & 928 \\
\hline 49981 & $5 / 5$ & 90 & 350 & 16,496 & 928 \\
\hline
\end{tabular}

Abbreviations: ATCC, the American Type Culture Collection; NG-MAST, Neisseria gonorrhoeae multiantigen sequence typing; PubMLST, public multilocus sequence typing; ST, sequence type. *Total number of successful/attempted cultures. DNA extraction and any subsequent procedures were individually performed for all the isolated colonies from each loop to confirm reproducibility. ${ }^{\dagger}$ The sequence was not found in the database; however, it shared $99 \%$ similarity with Allele $8442 .{ }^{*}$ There was a $1.2 \%$ mismatch of porB sequence with Allele 958. out of five cultures (Table 1). The overall recovery rate was $87.5 \%$.

\section{Sequence type and allele number determination by NG-MAST and PubMLST}

The results of NG-MAST and porB sequence typing using PubMLST are presented in Table 1. ATCC 19424 was identified as ST 266 by NG-MAST, and as Allele 946 by PubMLST. ATCC 31426 was identified as a novel ST by NG-MAST and assigned Allele 958 with a $1.2 \%$ mismatch by PubMLST. ATCC 35541 was identified as ST 12 by NG-MAST, and as Allele 624 by PubMLST. ATCC 43069 and ATCC 43070 were both identified as ST 681 by NG-MAST, and as Allele 984 by PubMLST. ATCC 49226 was identified as ST 1572 by NG-MAST, and as Allele 2110 by PubMLST. ATCC 49926 and ATCC 49981 were both identified as ST 16496 by NG-MAST, and as Allele 928 by PubMLST.

\section{DISCUSSION}

All the analysed ATCC reference strains, except for ATCC 31426, were identified as previously known types by both NG-MAST and PubMLST. For ATCC 31426, which was the only $\beta$-lactamase-positive strain among the eight strains in this study, the porB sequence was assigned Allele 958 with 98.8\% similarity by PubMLST, and the combination of Allele 8442 for porB and Allele 362 for $t b p B$ resulted in it being identified as a novel ST by NG-MAST.

ATCC 43069 and ATCC 43070 were identified as the same type by both NG-MAST and PubMLST. According to information provided by the ATCC website (https:// www.atcc.org), ATCC 43069 and ATCC 43070 were deposited by the same individual. There is a possibility that the two strains share the same origin. ATCC 49926 and ATCC 49981 were also identified as the same type by both NG-MAST and PubMLST; however, in this case, the information provided by the ATCC was insufficient to infer whether they have the same origin or not. The phylogenetic analysis of por $B$ and $t b p B$ was also in agreement with the NG-MAST and PubMLST results for 


\section{Journal of LABORATORY MEDICINE and QUALITY ASSURANCE}

Yousun Chung et al • Molecular Typing of ATCC N. gonorrhoeae

A

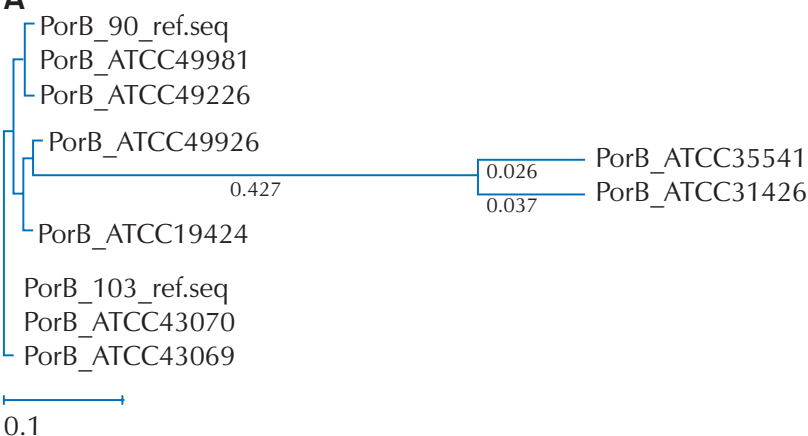

B

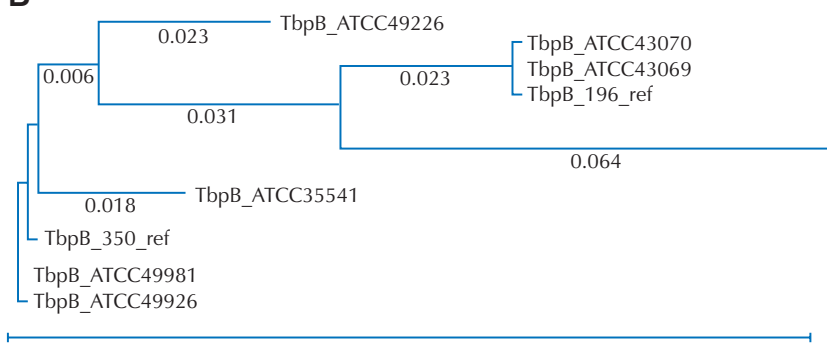

0.1
Fig. 1. Phylogenetic analysis of the por $B$ and $t b p B$ gene sequences. Abbreviation: ATCC, the American Type Culture Collection.
ATCC 43069 and ATCC 43070, and for ATCC 49926 and ATCC 49981 (Fig. 1).

We used five individually packaged Culti-Loops (Thermo Fisher Scientific) for each reference strain to verify the reproducibility of the test results. Although colonies were not obtained from some cultures, we confirmed that the por $B$ and $t b p B$ sequences, and the resulting NG-MAST and PubMLST type identifications, were the same during the repeated analyses. Culti-Loops are disposable bacteriological loops containing stabilised, viable microorganisms in a gel matrix, and have complete traceability, with ATCC licence [19]. Although the loops were stored and cultured as instructed by the manufacturer, the recovery rate was $87.5 \%$. Increasing the recovery rate of Culti-Loops should be addressed to facilitate their applicability in clinical microbiological laboratories.

Continuous monitoring of gonococcal strains by molecular sequencing analysis is crucial for determining trends in epidemiological change and bacterial resistance. Reference strains are especially useful for laboratory method quality assurance in epidemiological surveillance. Since NG-MAST and PubMLST are online public databases being adopted for molecular typing globally, in the current study, we genetically characterised eight ATCC gonococcal strains using these databases. To the best of our knowledge, analysis of these ATCC gonococcal reference strains by NG-MAST and PubMLST had not been reported before. The data obtained in this study can be used for quality assurance of molecular epidemiological typing in clinical microbiological laboratories.

\section{ACKNOWLEDGMENTS}

This work was supported by the Korean Ministry of Trade, Industry and Energy (Technology Innovation Program 10047748).

\section{REFERENCES}

1. Newman L, Rowley J, Vander Hoorn S, Wijesooriya NS, Unemo M, Low N, et al. Global estimates of the prevalence and incidence of four curable sexually transmitted infections in 2012 based on systematic review and global reporting. PLoS One 2015;10:e0143304.

2. World Health Organization. WHO guidelines for the treatment of Neisseria gonorrhoeae, 2016. http://apps. who.int/iris/bitstream/handle/10665/246114/978924154 


\section{Journal of LABORATORY MEDICINE and QUALITY ASSURANCE}

Yousun Chung et al • Molecular Typing of ATCC N. gonorrhoeae

9691-eng.pdf;jsessionid=C5B721118B62751056B9B516E 157858D? sequence=1 (Accessed December 17, 2018).

3. Centers for Disease Control and Prevention. Sexually Transmitted Disease Surveillance, 2016: gonorrhea statistics. https://www.cdc.gov/std/stats16/Gonorrhea.htm (Accessed December 17, 2018).

4. Unemo M, Dillon JA. Review and international recommendation of methods for typing neisseria gonorrhoeae isolates and their implications for improved knowledge of gonococcal epidemiology, treatment, and biology. Clin Microbiol Rev 2011;24:447-58.

5. Martin IM, Ison CA, Aanensen DM, Fenton KA, Spratt BG. Rapid sequence-based identification of gonococcal transmission clusters in a large metropolitan area. J Infect Dis 2004;189:1497-505.

6. Thakur SD, Levett PN, Horsman GB, Dillon JA. Molecular epidemiology of Neisseria gonorrhoeae isolates from Saskatchewan, Canada: utility of NG-MAST in predicting antimicrobial susceptibility regionally. Sex Transm Infect 2014;90:297-302.

7. Thakur SD, Starnino S, Horsman GB, Levett PN, Dillon JR. Unique combined pen $\mathrm{A} / \mathrm{mtrR} /$ porB mutations and NG-MAST strain types associated with ceftriaxone and cefixime MIC increases in a 'susceptible' Neisseria gonorrhoeae population. J Antimicrob Chemother 2014;69:1510-6.

8. Lebedzeu F, Golparian D, Titov L, Pankratava N, Glazkova S, Shimanskaya I, et al. Antimicrobial susceptibility/ resistance and NG-MAST characterisation of Neisseria gonorrhoeae in Belarus, Eastern Europe, 2010-2013. BMC Infect Dis 2015;15:29.

9. Jabeen K, Bhawan Mal P, Khan E, Chandio S, Jacobsson S, Unemo M. Antimicrobial resistance and Neisseria gonorrhoeae multiantigen sequence typing (NG-MAST) genotypes in N. gonorrhoeae during 2012-2014 in Karachi, Pakistan. BMC Infect Dis 2016;16:353.

10. Chen SC, Yin YP, Chen XS. Cephalosporin-resistant Neisseria gonorrhoeae clone, China. Emerg Infect Dis 2018;24:804-6.

11. Al Suwayyid BA, Coombs GW, Speers DJ, Pearson J, Wise
MJ, Kahler CM. Genomic epidemiology and population structure of Neisseria gonorrhoeae from remote highly endemic Western Australian populations. BMC Genomics 2018;19:165.

12. Maiden MC, Bygraves JA, Feil E, Morelli G, Russell JE, Urwin R, et al. Multilocus sequence typing: a portable approach to the identification of clones within populations of pathogenic microorganisms. Proc Natl Acad Sci U S A 1998;95:3140-5.

13. Harrison OB, Clemence M, Dillard JP, Tang CM, Trees D, Grad YH, et al. Genomic analyses of Neisseria gonorrhoeae reveal an association of the gonococcal genetic island with antimicrobial resistance. J Infect 2016;73:57887.

14. Lewis DA, Sriruttan C, Muller EE, Golparian D, Gumede L, Fick D, et al. Phenotypic and genetic characterization of the first two cases of extended-spectrum-cephalosporin-resistant Neisseria gonorrhoeae infection in South Africa and association with cefixime treatment failure. J Antimicrob Chemother 2013;68:1267-70.

15. Bennett JS, Jolley KA, Sparling PF, Saunders NJ, Hart CA, Feavers IM, et al. Species status of Neisseria gonorrhoeae: evolutionary and epidemiological inferences from multilocus sequence typing. BMC Biol 2007;5:35.

16. Golparian D, Dona V, Sanchez-Buso L, Foerster S, Harris $S$, Endimiani A, et al. Antimicrobial resistance prediction and phylogenetic analysis of Neisseria gonorrhoeae isolates using the Oxford Nanopore MinION sequencer. Sci Rep 2018;8:17596.

17. Lahra MM, Martin I, Demczuk W, Jennison AV, Lee KI, Nakayama SI, et al. Cooperative recognition of internationally disseminated ceftriaxone-resistant Neisseria gonorrhoeae strain. Emerg Infect Dis 2018;24. https://doi. org/10.3201/eid2404.171873.

18. Clinical and Laboratory Standards Institute. Performance standards for antimicrobial susceptibility testing: CLSI document M100S. 26th ed. Wayne (PA): Clinical and Laboratory Standards Institute, 2016.

19. Thermo Fisher Scientific. Package insert for Culti-Loops. Waltham (MA): Thermo Fisher Scientific, 2012. 